\title{
The Role of Imaging Informatics in Disaster Preparedness During the COVID-19 Pandemic
}

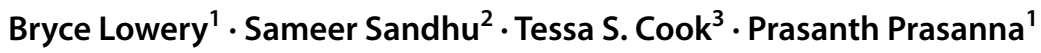

Accepted: 20 May 2021 / Published online: 23 June 2021

(c) Society for Imaging Informatics in Medicine 2021

\begin{abstract}
Disaster preparedness is a major but necessary undertaking for every health care facility. The 2019 coronavirus (SARSCoV-2) provided an unforeseen opportunity to compare the response of two radiology departments, University Health System A (UHSA) and University Health System B (UHSAB). Preparing for this disaster was unique since though unexpected, was thought to be detected early enough to allow for sufficient preparation. Unlike many other disasters which are short-term, single events, this has been an on-going event. Changes at both health systems included workflow alterations for exposure reduction to faculty, trainees, and staff. UHSA was able to quickly divert workflow to previously deployed home workstations, while University of Utah Health Sciences Center required 2 to 3 weeks to procure and initialize enough remote workstations to significantly affect departmental operations. Other measures such as universal masking, temperature screening at facility entrances, virtual appointments, and physical barriers were used by both systems to limit patient-to-patient, patient-to-staff, staff-to-patient, and staff-staff physical interaction to help decrease exposure risk. The goal of these preparations is to allow each department to fulfill imaging needs in support of the organizational clinical mission with the flexibility to adapt to the unique and dynamic nature of this disaster.
\end{abstract}

\section{Introduction}

In November 2019, an outbreak caused by severe acute respiratory syndrome coronavirus 2 (SARS-CoV-2) first identified in Wuhan, China, was declared to be a Public Health Emergency of International Concern by the World Health Organization in January 2020. Subsequently, the infection spread rapidly throughout the globe, and coronavirus disease 2019 (COVID-19) was recognized as a pandemic in March 2020. As of June 18, 2020, approximately 8.41 million cases of COVID have been reported in 188 countries resulting in approximately 450,000 deaths [1].

Initially, most radiologists focused on the role of imaging in diagnosing COVID-19 [2]. However, as understanding of

Prasanth Prasanna

prasanth45@gmail.com

1 Department of Radiology and Imaging Sciences, University of Utah Health Sciences Center, Salt Lake City, UT, USA

2 Department of Radiology, Hospital of University of Pennsylvania, Philadelphia, PA, USA

3 Perelman School of Medicine, University of Pennsylvania, 3400 Spruce Street, 1 Silverstein Radiology, Philadelphia, PA 19104, USA the disease process grew, they began to recognize that radiographic and CT findings must be evaluated together with the clinical presentation and additional diagnostic tests such as reverse transcriptase-polymerase chain reaction (RT-PCR) for viral RNA to confidently diagnose COVID-19. In parallel, most radiology practices also began to focus not only on diagnostic capability but also disaster preparedness. Many radiology practices have previously prepared for disasters such as mass casualty incidents [3], terrorist attacks [4], or natural disasters [5]. In addition, radiology departments have prepared for prior public health emergencies such as the Ebola [6] and SARS outbreaks [7]. However, many of these incidents were single or short-term events, unlike the current COVID-19 pandemic which may last months to years. Since it will impact radiology workflow for some time, this new challenge warrants a more long-term strategic response, effectively transitioning from the acute disaster response into business continuity practices.

Radiology preparedness encompasses a set of procedures/ policies implemented to enable practices to best continue clinical operations during a healthcare emergency [8]. Given the nature of the work and the ways in which care is delivered by radiology, imaging informatics plays a significant role in disaster preparedness. In this paper, we discuss the 
measures that two academic institutions, the University of Pennsylvania Health System (UHSA) and the University of Utah Health Sciences Center (UHSB), have taken to help combat this public health disaster.

Please note that the measures discussed in this paper reflect the recommendations and accommodations in place at these two institutions at the time of this manuscript submission. While some of these recommendations will still be valid, others will likely have evolved as more is learned about COVID-19.

The total numbers of employees at UHSA at the time of intervention include 152 full-time radiologists, 11 parttime radiologists, 8 divisions through the department, 75 residents (2020-21), 32 fellows (2020-21), 662 technical staff, and 63 nurses. Enterprise IT staff radiology in addition to other imaging departments and changes to the workflow were made in consultation between enterprise IT and Radiology department leadership.

The total numbers of employees at UHSB at the time of intervention include 60 full-time radiologists, approximately 10 part-time radiologists, 8 divisions through the department, 31 residents (2020-2021), 19 fellows (2020-2021), 210 technical staff, and 40 nurses. The IT/informatics team through which the majority of these changes were made is within the department of radiology. Hospital-wide changes were made with consultation of the department of radiology chair.

\section{Workflow Changes}

\section{Workstations}

There are multiple considerations when deploying remote workstations for radiologists, including technical specifications of the workstation computer and displays, reading location background lighting, network speed, and bandwidth. Home workstation monitors, like any other hospital workstation, should meet requirements for diagnostic image interpretation, including resolution and brightness levels $[9,10]$. Software should be configured by the practice to ensure compatibility with PACS, the electronic medical record (EMR), and any other required software. Multi-site practices that use more than one PACS or EMR may need to deploy more than one workstation if the different systems have incompatible software dependencies. Residential internet speed and internet service provider (ISP) data caps may require radiologists practicing remotely to change their ISP or internet coverage plan. In addition, arrangements should be made for technical support for home workstations in conjunction with the IT department, and logistical factors such as VPN expansion to handle increased traffic, delivery, potential need for in-person installation/service, and possible replacement of home workstations should also be considered. Of note, mammography was generally excluded from the discussion of home workstations due to the hardware technical requirements and cost. Mammography division essential operations were performed on-site, while nonessential operations were delayed or rescheduled.

As the number of COVID-19 cases increased, both departments started shifting daytime radiologists out of the reading room to home workstations. At UHSA, most of the faculty already had home workstations deployed before the pandemic. Prior to COVID-19, these workstations were mainly used to provide 24/7/365 backup to on-call residents and fellows, as well as to over-read cases on weekends. Virtually none of the divisions in the department had radiologists regularly working their day shifts from home. However, as the severity of the pandemic grew, UHSA radiologists were quickly able to respond to the need for decreased population density in the reading rooms by working daytime shifts from home. This also enabled the department to repurpose some reading room workstations as additional home-reading setups for residents and fellows. Residents and fellows considered at high risk for complications from COVID-19, or who could not easily commute to work (since public transportation completely shut down or had significant service reduction) were prioritized. Ultimately, nearly half of the UHSA radiology residents were able to work from home.

At UHSB, home workstations were rare within the system prior to COVID-19, with only two offsite adjunct faculty having home workstations out of approximately 60 full-time equivalent faculty. Early in the course of preparations, an urgent need for home workstations was realized and equipment acquisition funding avenues were explored. Foreseeing supply-chain erosion, equipment was quickly ordered utilizing department emergency funds. Prior to delivery, transferring underutilized workstations to radiologists' homes was initially considered. Fortunately, the new equipment arrived quickly, and this contingency plan was not implemented. Over about 3 weeks, home workstation deployment progressed from 2 to 18 which allowed a reduction in the number of on-site radiologists to 1 to 2 radiologists per division per weekday and 0 to 1 per division for weekends and after-hours staffing. Some necessary equipment, such as non-diagnostic workstation monitors, though back-ordered slowly started to arrive after about 2 weeks. Priority for home workstations went to individuals who were quarantined or self disclosed as high-risk for COVID-19. This was followed by those experiencing significant family care difficulties secondary to school and childcare closures. Remaining workstations were then prioritized to ensure that each division could be covered from at least one home workstation. A total of about 40 to 60 operable workstations were planned to facilitate a fully functioning department with the 
majority of image interpretation performed remotely, and prepare for the possibility that a large portion of the department may end up under quarantine.

At UHSB, the decision was made for home workstations to either be picked up by the user, or if unable to do so due to quarantine, the machine would be delivered outside the user's home. This was to help reduce the possibility of contamination for either radiologist or the PACS/IT team member. Setup instruction booklets were created, and IT staff were available by phone or video conference to provide additional support. Most radiologists only encountered minor difficulties in setting up their home workstations. In total, 47 of the desired 40 to 60 workstations were eventually operational which did not allow for full offsite departmental operations, but significantly aided in reducing on-site staffing. UHSB also created its own in-house ticketing system, launched via a PACS plugin, that could be used for PACS/ IT support.

\section{Reading Room Adaptations}

At UHSA, measures were taken to decrease in-person interaction among the staff where possible. One of the easiest changes was decreasing the number of faculty and trainees in the reading room simultaneously, since as previously discussed most of the faculty already had home workstations. Creative approaches were implemented to enable residents to run PACS, access the EMR, and dictate cases with voice recognition software using personal computing hardware already in their homes. Residents who did not already have remote access via the virtual private network (VPN) were granted emergency exemptions. A few workstations were also redeployed from reading rooms to radiologists' homes, in lieu of waiting on new equipment arrival. Call shifts were adjusted to minimize radiologist concentrations in the reading room; again, faculty were encouraged to read remotely on call when possible.

Ancillary spaces within UHSA were also adapted to enable in-house radiologists to work while physically distanced. Technologists from the 3-D and Advanced Imaging Laboratory, which provides real-time post-processing services for clinical cases, were redeployed offsite using laptops already in possession for weekend and off-hours coverage, [11]. The Lab provided space for an additional 2 radiologist workstations that could be used for overflow. Workstations at outpatient imaging centers were another potential space for radiologists who lived close by, particularly when patient volumes at those locations were low.

The strategy to maximize physical distancing at UHSB employed several similar techniques. Two isolated reading rooms were setup, and each division managed staffing to ensure as little exposure as necessary. With the reduction in clinical volumes, plans to open more isolated reading rooms were created to bolster the number of reading locations already scattered throughout the clinical facilities. Additionally, workstations were assigned and labeled for use by $1-2$ radiologists only.

\section{Clinical Coverage}

At UHSA, the frequency of staff rotating between services was decreased for both trainees and faculty, both to limit exposure and form teams that would work together but not compromise an entire division by exposing multiple members of the department. The Division of Interventional Radiology developed non-overlapping teams of radiologists, technologists, and nurses. Some of the diagnostic divisions modified their schedules to pair trainees and faculty together, so the same individuals were in the same reading rooms, using the same workstations each time.

The measures taken to optimize clinical coverage at UHSB similarly involved some divisions dividing their staff members into different teams. For instance, Team A would work one day, then Team B the next day, then Team C, etc., with rotation continuing to minimize contact between the teams. Staff not covering the clinical service was scheduled for academic time. The musculoskeletal division utilized a graduated approach, by quickly decreasing staffing as volume decreased and eventually rotating through a single attending, single fellow, and a single resident per day. Topics of staffing discussion initially included tailoring the rotation around subspecialty interpretation, such as musculoskeletal ultrasound, in which only a few providers are proficient. This would have dictated the maximum number of independent teams possible for the division, though this strategy became unnecessary once all elective procedures were postponed.

Both institutions also provide some radiology services to their respective Veterans' Affairs (VA) Hospitals. At UHSB, this created challenges in coverage, as VA studies could only be read either at three workstations in the university hospital or at the VA itself. As members of our on-site staff were reduced, either due to acquisition of home workstations or rotation frequency, similar adjustments for appropriate VA coverage were made. For UHSA diagnostic radiologists who most frequently cared for VA patients, a handful of home workstations were made available. Radiologists who covered VA patients less frequently continued to read as part of a skeleton crew in the reading room, and observed appropriate physical distancing since fewer than half of the workstations in the room were occupied.

At both institutions, the pandemic led to a projected exponential increase in chest imaging and a decrease in imaging volume in the majority of the remainder of the department. Therefore, changes were made to the workflow to increase off-hours faculty coverage on the cardiothoracic service. Additionally, faculty and trainees from other divisions were 
assigned to cover the cardiothoracic imaging divisions at both institutions. In addition, many fellows with unrestricted medical licenses (who are credentialed to read chest radiographs and chest CTs independently) could have been assigned to the cardiothoracic service if the volume of exams had increased.

With the growing need for staff in other parts of the hospital, such as the emergency room (ER), inpatient wards, intensive care units (ICUs), and ambulatory surge clinics, both radiology departments were asked to prepare to redeploy their staff if needed. At UHSA, a committee was formed by the Workforce Resource Center (which works with department chairs) to discuss how both trainees and faculty could be reassigned based on the skill sets that best match clinical needs. Specifically, trainees and faculty could have been assigned to help in an outpatient COVID-19 testing site, screening/testing centers, and with low-risk ER patients. Interventional radiologists and first-year residents with more recent hands-on patient care experience could potentially have been redeployed to an ICU or in-patient floors. Additional considerations prior to redeployment of a staff member to activities that may have required direct care of COVID-19 positive patients included any personal health risks, family health risks, age, pregnancy status, and child care needs.

Redeployment was also considered possible at UHSB, and a survey was sent to evaluate and estimate the number of available employees that would be able to assist in certain areas. Although these contingencies were put in place early by both health systems, they were ultimately not activated. Had the need arisen, and the anticipated surge in chest imaging also materialized, the radiology departments would have activated a staffing plan to prioritize coverage of departmental services while still attempting to support the staffing needs elsewhere in their health systems.

\section{Image Acquisition}

At UHSB, a few new processes were introduced for how orders and examinations were handled in the radiology department. First, a "Person Under Investigation" (PUI) exam type was employed for both chest $\mathrm{X}$-rays and chest CTs within our EMR and PACS. This is in concordance with the CDC recommendations to categorize a patient who is being evaluated for COVID-19. Transportation routes and care facilities also needed to be taken adjusted as temporary outdoor tents near the Emergency Department handled some of the patient overflow.

A novel technique for performing portable chest imaging was implemented at UHSB to allow technologists to refrain from entering the patient's negative pressure room (Fig. 1). The technique involved moving the detector plate into the room but keeping the X-ray generation source outside the room. This technique was successfully altered to optimize images without a significant loss in quality.

At UHSA, the PUI designation was also used and sometimes included in the order history for the patient. The image acquisition workflow was modified to add portable X-ray machines to support the outdoor screening tents installed at the ER entrances throughout the health system.

\section{Structured Reporting}

The cardiothoracic radiologists at UHSA collaborated with others across the country to develop guidelines for the appropriate use of CT for imaging patients with known or suspected disease, and an accompanying structured reporting template, [12]. Locally, at UHSA, standardized templates were developed for both chest radiography (Table 1) and chest CT (Table 2) in PUIs for COVID-19. The chest radiograph template allowed the radiologist to describe the findings and then add a label to the report indicating the degree of suspicion for pneumonia (not specifically COVID19 pneumonia). The chest CT template similarly allowed for a description of the findings in the lungs, accompanied by structured language describing the likelihood of COVID-19 infection given the imaging appearance. These templates not only serve to standardize the language used to report these examinations, but also to reduce variability in the interpretation of CT reports by clinicians. In addition, they enable radiologists to create labeled data in the course of routine clinical practice, which can then be used for research, and ultimately, AI development or evaluation. Radiologists and trainees in the department were educated on the use of the template via a series of emails, and a detailed slide deck with examples of the imaging findings intended to prompt a particular choice in the template.

At UHSB, a similar approach to reporting was implemented for chest radiographs and chest CTs to facilitate communication with clinicians given the unfamiliar new workflows, including the need to be thorough without utilizing excess resources.

\section{Resident and Fellow Education, Conferences, and Meetings}

At UHSA, all large staff gatherings, including resident/fellow conferences, grand rounds, and other multidisciplinary conferences, were virtualized and conducted via web conference, using an existing enterprise-wide solution that supported both smaller meetings and larger events. The larger events were analogous to webinars, allowing for a designated moderator to manage participants and a dedicated questionand-answer feature. Accounts to be used for telehealth encounters with patients were also created after the CMS exemption for telehealth, [13]. 
Fig. 1 Portable X-ray configuration at UHSB used to protect $\mathrm{X}$-ray technologists taking care of patients under investigation for COVID-19

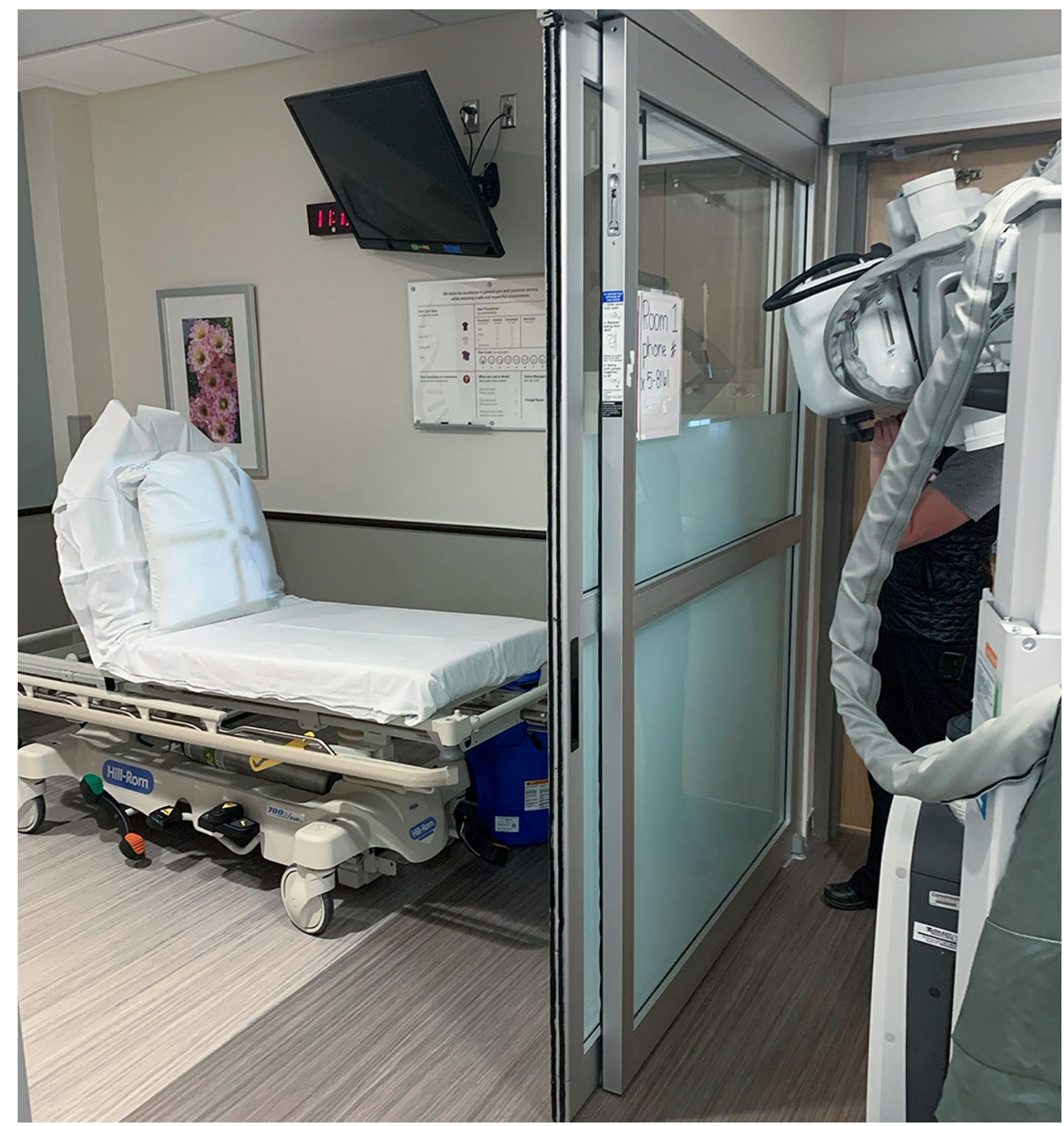

At UHSB, all non-urgent radiology section and departmental meetings were canceled, postponed, or moved to the virtual setting. UHSA and UHSB conducted at least one inter-institutional resident conference during this time, which was facilitated by the fact that all resident education had suddenly been virtualized. The presence of the new home workstations at UHSB aided the creation of new lecture material as well.

Table 1 Summary of the template for reporting chest radiography in patients under investigation for COVID-19 developed at UHSA

\begin{tabular}{ll}
\hline Text label & Template text \\
\hline XR_NP & Negative for pneumonia \\
XR_IP & Nonspecific and \\
& indeterminate for \\
& pneumonia \\
XR_FP & Focal pneumonia \\
XR_MP & Multifocal pneumonia
\end{tabular}

To continue to deliver high-quality trainee education, virtual didactic and case-based conferences were added to offset the decrease in case volume and in-person teaching throughout the department, [14, 15]. Resident readouts during the day were conducted through a combination of web conferencing to enable image sharing and simultaneous review of the same images. In addition, the native chat

Table 2 Summary of the template for reporting chest CT in patients under investigation for COVID-19 developed at UHSA. This formed the basis for the templates that were ultimately included in the RSNA Consensus Statement [12]

\begin{tabular}{ll}
\hline Text Label & Template Text \\
\hline Co_Lo & Low confidence for COVID-19 pneumonia \\
Co_In & Intermediate confidence for COVID-19 pneumonia \\
Co_Hi & High confidence for COVID-19 pneumonia \\
Co_Ad & $\begin{array}{c}\text { Unlikely to represent COVID-19 pneumonia; } \\
\text { alternative diagnosis favored }\end{array}$ \\
Co_Neg & No COVID-19 pneumonia \\
\hline
\end{tabular}


feature in PACS became even more widely used as a means to provide feedback to residents and enable communication between radiologists and trainees who were not in the same reading room.

During times of uncertainty, communication between the leadership and its workforce is crucial. At UHSA, the radiology department chair held weekly virtual town hall sessions to discuss policy and staffing changes, as well as provide updates on departmental and organizational progress in combating the pandemic. The chairman has been sending a daily email to the department providing data on the trend in weekly/daily infection rate, inpatient admissions, and ICU admissions from COVID-19. Other information, such as resources for child care, were discussed.

The UHSB department chair took a similar approach, using department-wide virtual meetings to communicate. Although these were initially held twice a week, they subsequently became weekly "huddles" to discuss trends of COVID-19 infection as well as policy and staffing changes. In addition, child care and mental health resources were also shared. The chairperson also sent out regular emails to the department with extra information and links to updated policies.

\section{Infection Control}

At the beginning of the pandemic, both organizations focused heavily on acquiring sufficient personal protective equipment (PPE) for their employees and allocating them judiciously, and in accordance with CDC guidelines. Subsequently, both organizations began contactless temperature screening for both employees and patients entering health system locations. Designated employee and patient entrances were identified and staffed.

Employees were required to wear surgical masks at all times within the hospital, and to don and doff additional PPE (including eye protection, face shields, and respirator masks/devices) if caring for PUIs or COVID-19 patients. As imaging is an essential part of healthcare today, these guidelines were also followed by radiology technologists. Procedures for cleaning imaging equipment for PUIs, confirmed COVID-19 patients, COVID-19 patients on ventilators, and after potentially aerosol-generating image-guided procedures were published on the UHSA COVID-19 website. UHSB published a decision tree of when to report to work or call in sick when feeling unwell, to help decrease the likelihood of understaffing.

Prior to COVID-19, CT scanners were already surface decontaminated between patients on contact precautions, and this practice was continued with confirmed or suspected COVID-19 patients. During the pandemic, after intubation or other aerosol-generating procedure within the CT suite, the room was closed for $60 \mathrm{~min}$ to allow room air turnover, followed by surface decontaminated, then re-opened for imaging. At UHSA, an ultraviolet tower was also used to clean the $\mathrm{CT}$ room or $\mathrm{CT}$ procedure suite after imaging or procedures on COVID-19 patients.

\section{Conclusions}

Unlike prior public health emergencies, the COVID-19 pandemic has required radiology practices to not only mount an immediate disaster recovery-type response, but also multiple contingencies and adaptations to business continuity policies as they continue to deliver care to patients with and without COVID-19. Radiology practices must be able to balance the safety and well-being of both patients and health care workers while delivering optimal imaging in alignment with the institutional clinical mission. Given the dynamic and ongoing nature of this pandemic, organizations need to be flexible and adapt their strategic response as new information about the virus is collected. Notably, the use of information technology and remote work to completely replace the work of onsite diagnostic radiologists would fall far short of clinician expectations since procedures are necessary on-site, and often one-on-one discussion leads to more effective communication, especially in the setting of subspecialty divisions that are commonplace among academic institutions. Although the discussion here was limited to two academic radiology departments, we hope the recommendations in this paper can provide a framework for any radiology practice to implement or revise their disaster preparedness measures during the current COVID-19 pandemic and future challenges to routine patient care.

\section{References}

1. Coronavirus COVID-19 Global Cases by the Center for Systems Science and Engineering (CSSE) at Johns Hopkins University (JHU). Baltimore, MD. https://gisanddata.maps.arcgis.com/apps/ opsdashboard/index.html\#/bda7594740fd40299423467b48e9ecf6

2. Radiology Perspective of Coronavirus Disease 2019 (COVID-19): Lessons From Severe Acute Respiratory Syndrome and Middle East Respiratory Syndrome Melina Hosseiny, Soheil Kooraki, Ali Gholamrezanezhad, Sravanthi Reddy, and Lee Myers American Journal of Roentgenology. 2020;214:1078-1082. https://doi.org/ 10.2214/AJR.20.22969

3. Emergency imaging after a mass casualty incident: role of the radiology department during training for and activation of a disaster management plan Ferco H Berger, Markus Körner, Mark P Bernstein, Aaron D Sodickson, Ludo F Beenen, Patrick D McLaughlin, Digna R Kool, and Ronald M Bilow. The British Journal of Radiology 2016 89:1061

4. Terrorist Attack in Nice, France, in July 2016: Massive Influx of Patients to a Radiology Department. Amoretti N. Radiology. May 15,2018

5. Heacock L, Tulin-Silver S. After the storm: eight unexpected benefits to radiology resident education at NYU Langone Medical Center after Hurricane Sandy. J Am Coll Radiol. 2014;11(1):97-100. https://doi.org/10.1016/j.jacr.2013.08.020 
6. Ebola Virus Disease: Radiology Preparedness.David A. Bluemke and Carolyn C. Meltzer. Radiology 2015 274:2, 527-531

7. Severe Acute Respiratory Syndrome: Management and Reconfiguration of a Radiology Department in an Infectious Disease Situation Ian Y. Y. Tsou, Jeffrey S. K. Goh, Gregory J. L. Kaw, and Thomas S. G. Chee Radiology 2003 229:1, 21-26

8. Radiology Department Preparedness for COVID-19: Radiology Scientific Expert Panel Mahmud Mossa-Basha, Carolyn C. Meltzer, Danny C Kim, Michael J Tuite, K. Pallav Kolli, Bien Soo Tan. Mar 16, 2020. https://pubs.rsna.org/doi/full/10.1148/radiol.20202 00988

9. Steckel, R.J., Batra, P., Goldin, J.G. et al. Supervision of residents by faculty radiologists using home workstations. Emergency Radiology 10, 121-125 (2003). https://doi.org/10.1007/ s10140-003-0283-6

10. ACR-AAPM-SIIM Technical Standars for Electronic Practice of Medical Imaging. Revised 2017. https://www.acr.org/-/media/ ACR/Files/Practice-Parameters/elec-practice-medimag.pdf

11. Cook TS, Steingall SJ, Steingall SR, Boonn WW. Establishing and Running a Three-dimensional and Advanced Imaging Laboratory. Radiographics. 2018;38(6):1799-1809. https://doi.org/10.1148/rg. 2018180058

12. Simpson S, Kay FU, Abbara S, et al. "Radiological Society of North America Expert Consensus Statement on Reporting Chest
CT Findings Related to COVID-19. Endorsed by the Society of Thoracic Radiology, the American College of Radiology, and RSNA". Radiology: Cardiothoracic Imaging 2(2), 2020. https:// pubs.rsna.org/doi/10.1148/ryct.2020200152

13. COVID-19 Emergency Declaration Blanket Waivers for Health Care Providers. 2020. https://www.cms.gov/files/document/ summary-covid-19-emergency-declaration-waivers.pdf. Accessed $5 / 2020$

14. Acad Radiol. 2020 May 11 https://doi.org/10.1016/j.acra.2020.05. 001 [Epub ahead of print] Radiology Residency Program Management in the COVID Era - Strategy and Reality Eric England, MD, a, Alisa Kanfi, MD, a Carl Flink, MD, a Achala Vagal, MD, MS, a David Sarkany, MD,b Maitray D. Patel, MD,c James Milburn, MD, FACR,d Seetharam Chadalavada, MD, MS, a and Sheryl Jordan, MD, RCCe

15. Radiology Residency Preparedness and Response to the COVID19 Pandemic Alice Chong MD, MHCIa Nolan J. Kagetsu MDb Andrew Yen MDa Erin A. Cooke MDc https://www.sciencedirect. com/science/article/pii/S1076633220301938Volume 27, Issue 6, June 2020, Pages 856-861

Publisher's Note Springer Nature remains neutral with regard to jurisdictional claims in published maps and institutional affiliations. 